Herausgeber:

Prof. Dr. Klaus Boers, Universität Münster | Prof. Dr. Heinz Cornel, Alice Salomon-FH Berlin | Prof. Dr. Frieder Dünkel, Universität Greifswald | Prof. Dr. Monika Frommel, Universität Kiel | Prof. Dr. Wolfgang Heinz, Universität Konstanz | Prof. Gabriele Kawamura-Reindl, Georg-SimonOhm-FH Nürnberg | Prof. Dr. Joachim Kersten, Deutsche Hochschule der Polizei, Münster | Prof. Dr. Karl-Ludwig Kunz, Institut für Strafrecht und Kriminologie, Bern | Prof. Dr. Heribert Ostendorf, Forschungsstelle für Jugendstrafrecht und Kriminalprävention an der Universität Kiel | Prof. Dr. Bernd-R. Sonnen, Universität Hamburg | PD Dr. Wolfgang Stangl, Institut für Rechts- und Kriminalsoziologie, Wien | Dr. Joachim Walter, ehemals JVA Adelsheim

\title{
Zum Referentenentwurf eines Gesetzes zur bundesrecht- lichen Umsetzung des Abstandgebots im Recht der Siche- rungsverwahrung des Bundesministeriums der Justiz vom 9. November 2011
}

Heinz Cornel

Nachdem das Bundesverfassungsgericht in seinem Urteil vom 4.5.2011 (2 BvR 2365/09) die Vorschriften des StGB über die Unterbringung in der Sicherungsverwahrung für nicht mit dem Grundgesetz vereinbar erklärt und den Gesetzgebern im Bund und in den Ländern aufgegeben hatte, ein Gesamtkonzept der Sicherungsverwahrung zu entwickeln, das dem verfassungsrechtlichen „Abstandsgebot" Rechnung trägt, hat das Bundesministerium der Justiz nach zahlreichen Beratungen mit den Ländern am 9.November 2011 einen Referentenentwurf vorgelegt. Dieser soll einer ersten Bewertung unterzogen werden, wobei in diesem kurzen Beitrag zur ersten Orientierung nicht auf die grundsätzlichen kriminalpolitischen Positionen eingegangen wird. Die drei Eckpunkte dazu sind bekannt: Zum einen die seit mehr als 75 Jahren bestehende Sicherungsverwahrung, deren Anwendung in den letzten Jahren stark ausgeweitet wurde; zum zweiten die kriminalpolitische Kritik, die immer wieder die Abschaffung der Sicherungsverwahrung forderte und zum dritten die Tatsache, dass noch vor einem Jahr kaum jemand sich hätte vorstellen können, wie grundsätzlich das Recht und die Praxis der Sicherungsverwahrung in Deutschland neu zu regeln sei.

Zunächst sei darauf hingewiesen, dass es, entgegen zeitweise in der Öffentlichkeit diskutierten Plänen, bei dem Begriff Sicherungsverwahrung geblieben ist. Im Folgenden sollen einige zentrale Regelungen des Referentenentwurfs, der am 17. November den betroffenen Fachkreisen und Verbänden mit der Bitte um Stellungnahme übermittelt wurde, aufgegriffen und kommentiert werden.
In $\ 66 c$ wird die „Ausgestaltung der Unterbringung in der Sicherungsverwahrung und des vorherigen Strafvollzugs" eingefügt. Nach Abs. 1 erfolgt die Unterbringung in Einrichtungen, die

dem Untergebrachten auf der Grundlage einer umfassenden Behandlungsuntersuchung und eines regelmäßig fortzuschreibenden Vollzugsplans Betreuung anbieten,

andividuell und intensiv betreuen (Individualisierungs- und Intensivierungsgebot - BVerfGE Rn. 113), die Mitwirkungsbereitschaft des Sicherungsverwahrten wecken und fördern (Motivierungsgebot - Rn. 114), sowie psychiatrische, psycho- oder sozialtherapeutische Behandlungen enthalten,

zum Ziel haben, die Gefährlichkeit der Verwahrten für die Allgemeinheit so zu mindern, dass die Vollstreckung der Maßregel möglichst bald zur Bewährung ausgesetzt oder sie für erledigt erklärt werden kann,

eine Unterbringung gewährleisten, die den Untergebrachten so wenig wie möglich belastet und, soweit Sicherheitsbelange nicht entgegenstehen, den allgemeinen Lebensverhältnissen angepasst ist,

in der Regel vom Strafvollzug getrennt sind und sich in besonderen Gebäuden oder Abteilungen befinden (Trennungsgebot Rn.115), 
vollzugsöffnende Maßnahmen gewähren und Entlassungsvorbereitungen treffen, soweit nicht zwingende Gründe entgegenstehen, insbesondere die konkrete Gefahr besteht, dass sich der Untergebrachte dem Vollzug entziehen oder die Maßnahmen zur Begehung schwerer Straftaten missbrauchen wird und

Nachsorge in Kooperation mit staatlichen und freien Trägern ermöglichen.

Der Entwurf legt damit kriminalpolitisch die Grundlagen für eine neue Ausrichtung der Sicherungsverwahrung, wie sie vom BVerfG insbesondere als Mindestaspekte eines Gesamtkonzeptes gefordert wurden (Rn. 111-118). Das ist zweifellos kein Abschied von und auch keine wirkliche Einschränkung der Anwendung der Sicherungsverwahrung, die das BVerfG auch nicht gefordert hatte, aber hinsichtlich der Ausgestaltung der Unterbringung zeichnet sich der Referentenentwurf meines Erachten weitgehend durch Konsequenz und Stimmigkeit aus.

$\mathrm{Zu}$ begrüßen ist auch, dass der Referentenentwurf in $\ 66 \mathrm{c}$ Abs. 2 StGB-E eigene Vorgaben für den Vollzug der Strafhaft vorsieht, die der Unterbringung in der Sicherungsverwahrung vorangeht. Dies greift eine häufig geäußerte Kritik an der Situation der letzten Jahrzehnte auf. Es ist dem Täter schon im Strafvollzug eine individuelle und intensive Betreuung anzubieten mit dem Ziel, die Vollstreckung der Unterbringung entbehrlich zu machen. Hier spricht das Bundesverfassungsgericht vom ultima-ratio Prinzip (a.a.O. Rn. 112). Wenn dies wirklich konsequent umgesetzt wird, wäre das in der Tat eine Chance, die Anwendung der Sicherungsverwahrung zu reduzieren.

Konsequent setzt der Referentenentwurf durch die $\mathbb{S}$ 67c Abs. 1 Nr. 2 und 67d Abs. 2 Satz 2 StGB-E die Folgen bei Nichteinhaltung der bundesgesetzlichen Vorgaben durch die Praxis fest. Die nicht dem Abstandsgebot entsprechende Ausgestaltung des Vollzugs der Sicherungsverwahrung bzw. des Vollzugs der vorangehenden Strafhaft muss zur Entlassung aus der Unterbringung führen. Man darf gespannt sein, ob diese Formulierungen das Gesetzgebungsverfahren überleben und wie sie in der Praxis von den Gerichten gehandhabt werden.

Zwingend geboten war auch die Abschaffung der nachträglichen Sicherungsverwahrung nach $\ 7$ Abs. 2 JGG. Allerdings wird $\ 7$ Abs. 2 nun durch die Absätze 2 und 3 ersetzt, die die vorbehaltene Sicherungsverwahrung vorsehen und bei Verurteilten bis zum siebenundzwanzigsten Lebensjahr die Vollziehung der Jugendstrafe in einer sozialtherapeutischen Anstalt fordern, soweit nicht die Resozialisierung anders besser gefördert werden kann. Dass der Entwurf die nachträgliche Sicherungsverwahrung im JGG durch eine erweiterte Vorbehaltsmöglichkeit ersetzt, ähnlich der bereits zum 1. Januar 2011 in Kraft getretenen Regelung im allgemeinen Strafrecht, ist im Hinblick auf die Abschaffung der nachträglichen Sicherungsverwahrung positiv, im Übrigen aber kritisch zu bewerten, weil einerseits zu befürchten ist, dass von der Vorbehaltslösung inflationär Gebrauch gemacht werden wird, um alle Möglichkeiten offen zu halten und zum zweiten, weil dies Einfluss auf den Resozialisierungsprozess während des Vollzugs haben muss (Stellungnahme des DBH-Fachverbands vom 22. November 2010, in: Bewährungshilfe 2010, Heft 4 S.445).

Wer wird sich im Zuge einer Behandlung offenbaren, wenn er befürchten muss, dass dies zu einer Unterbringung in Sicherungsverwahrung führen kann? Die Kritik an der Vorbehaltsmöglichkeit für Ersttäter gilt erst recht für die nunmehr auch gegenüber Jugend- lichen ( $\mathbb{7}$ Abs. 2 JGG-E) und Heranwachsenden ( $\mathbb{S} 106$ Abs. 3 JGG-E) vorgesehene Möglichkeit. Reichen schon bei Erwachsenen die Erkenntnismöglichkeiten nach einer einzigen Tat nicht für den Vorbehalt einer so schwerwiegenden Sanktion aus, gilt das erst recht für Jugendliche und Heranwachsende.

$\mathrm{Zu}$ begrüßen ist, dass der Entwurf für diejenigen, die für nach dem Inkrafttreten der Neuregelung begangene Taten verurteilt werden, an der bereits zum 1. Januar 2011 getroffenen Entscheidung zur Abschaffung der nachträglichen Sicherungsverwahrung für künftige Fälle festhält. Diese Regelung wird sicher in der kriminalpolitischen Debatte über diesen Referentenentwurf eine ganz herausragende Rolle spielen. Man darf gespannt sein, ob dies so im Bundesgesetzblatt landen wird.

Durch Änderungen und Einfügungen in $\$ 463$ StPO ist für die rechtzeitige Bestellung eines Verteidigers bei Anordnung und Vollstreckung der Sicherungsverwahrung gesorgt. Dies ist ebenso zu begrüßen wie die zahlreichen gerichtlichen Überprüfungen des Vollzugs von Sicherungsverwahrung innerhalb z. T. gegenüber dem gegenwärtigen Zustand deutlich verkürzten Fristen (vgl. \$\$ 67d, e StGB-E sowie $\mathbb{S} 119$ a StVollzG-E; dies entspricht direkt den Forderungen des BVerfG im Sinne eines Rechtsschutz- und Unterstützungsgebots sowie Kontrollgebots - Rn. 117-118). Dadurch werden noch mehr als heute gerichtliche und anwaltliche Kosten entstehen, die der Entwurf im Einklang mit den bisherigen Grundsätzen dem Betroffenen auferlegt. Das wird, zumal unter den jetzt richtigerweise gesteigerten Anforderungen, zu einer so deutlichen finanziellen Belastung des Betroffenen führen, dass diese eine erfolgreiche Reintegration erschweren wird. Hier sollte der Gesetzgeber im Laufe des weiteren Verfahrens nachbessern und vor allem die finanziellen Belastungen durch die neu hinzukommenden Begutachtungen, gerichtlichen Verfahren etc. der Landeskasse zuweisen. Faktisch bedeutet das für die Länder eine relativ geringe Belastung, da sie bei den meist mittellosen Betroffenen die entsprechenden Kosten schon jetzt letztlich selbst tragen müssen. Es wäre aber für die Betroffenen ein durchaus sinnvolles und spürbares Zeichen, wenn ihre Aussichten auf Wiedereingliederung in dieser Hinsicht nicht weiter verschlechtert würden.

Im Übrigen wird sich die künftige Praxis der Länder daran messen lassen müssen, ob die Gerichte für die neuen Aufgaben personell gerüstet sind. Sollte sich diese gerichtliche Kontrolle in der Praxis der Sicherungsverwahrung bewähren, dann liegt es nahe, die Frage aufzuwerfen, ob diese Form bzw. das Verfahren nicht auch für länger dauernde zeitige oder lebenslange Freiheitsstrafen ohne anschließende Unterbringung vorzusehen ist.

Mit der Übergangsregelung des Art. 316f EGStGB-E schreibt der Entwurf die Fortgeltungsanordnung aus dem Urteil des BVerfG über den 31. Mai 2013 hinaus fort. Es bleibt also - mit der vom BVerfG vorgegebenen Beschränkung auf psychisch gestörte und deshalb hochgefährliche Straftäter - bei der Anordnungsmöglichkeit von nachträglicher Sicherungsverwahrung für Taten vor dem Inkrafttreten der Neuregelung. Der Bundesgesetzgeber nimmt sich da meines Erachtens großzügig Zeit und schadet damit der Zielsetzung. Das BVerfG hatte seine Fortgeltungsanordnung ausdrücklich mit der Sorge begründet, „dass die Funktionsfähigkeit des bestehenden zweispurigen deutschen Maßregel- und Strafrechtssystems nachhaltig gestört wäre. Alle in der Sicherungsverwahrung untergebrachten Personen müssten sofort freigelassen werden, was Gerichte, Verwaltung und Polizei vor kaum lösbare Probleme stellen würde“ (a.a.O. Rn. 169). Die Sorge galt also erkennbar der unmittelbar auf die Ent- 
scheidung folgenden Zeit, eben bis längstens zum Inkrafttreten einer Neuregelung, und selbst für diesen Zeitraum hat das Gericht die Anwendungsmöglichkeiten durch das bereits beschriebene zusätzliche Erfordernis einer psychischen Störung und einer besonders hochgradigen Gefährlichkeit beschränkt. Es wäre ohne weiteres möglich gewesen, für die Zeit nach Ablauf dieser Übergangsphase die Anordnung der nachträglichen Unterbringung generell auszuschließen, nicht erst für Taten, die nach Inkrafttreten der Neuregelung begangen werden. Die Begründung für diese Regelung des Referentenentwurfs schreibt dazu lapidar: „Damit wird ... diese rechtlich und tatsächlich problematische Anordnungsform noch so lange fortgeführt, bis der Schutz der Bevölkerung durch den Ausbau insbesondere der vorbehaltenen Sicherungsverwahrung übernommen werden kann“ (ReferentenE S. 16). Es bleibt abzuwarten, ob das Bundesverfassungsgericht damit zufrieden sein wird, ob das der geforderten ,Freiheitsorientierung' (BVerfGE a.a.O. Rn.120) entspricht, zumal die Begründung des Referentenentwurfs selbst formuliert, dass „die Fortgeltung der einschlägigen Regelungen über die vom Urteil des BVerfG erfasste Übergangszeit hinaus“ bestimmt werde.

Insgesamt greift der Referentenentwurf die Vorgaben des Bundesverfassungsgerichts weitgehend angemessen auf und setzt sie in bundesgesetzliche Zielvorgaben um. Manchmal hätte man sich weitergehende und konkretere Regelungen gewünscht, wobei man die Kompetenzverteilung nach der Föderalismusreform berücksichtigen muss, die Bundes- und Landesgesetzgeber zur Entwicklung eines Gesamtkonzepts verpflichtete. Man darf gespannt auf die Umsetzungen in den Ländern in den Gesetzgebungsverfahren, der Praxis der Gerichte und des Justizvollzugs sowie hinsichtlich der Verzahnungen mit den Sozialen Diensten der Justiz und den Freien Trägern sein. Wer sich auf die oben genannten Eckpunkte bezieht, der wird feststellen, dass der Referentenentwurf natürlich Forderungen zur Abschaffung der Sicherungsverwahrung nicht aufnimmt, aber dass er andererseits mehr an Rechtsstaatlichkeit und rationaler Kriminalpolitik beinhaltet, als dies die zurecht gerügte Praxis der Sicherungsverwahrung der letzten Jahrzehnte mit sich brachte. Und schließlich wird man bei aller hier geäußerten Kritik darauf hinweisen können, dass der jetzt vorliegende Referentenentwurf die Forderungen des Bundesverfassungsgerichts bis auf die Übergangsvorschriften ${ }^{1}$ uneingeschränkt berücksichtigt. Das Bundesverfassungsgericht hatte in seinem Urteil vom 4.Mai 2011 die Länder und den Bund verpflichtet, „ein freiheitsorientiertes und therapiegerichtetes Gesamtkonzept für die Unterbringung zu entwickeln “2 und dabei insb. dem Abstandsgebot zum Strafvollzug gerecht zu werden. Das sind große Worte, aber der Entwurf zeigt das Bemühen des Bundesjustizministeriums, die geforderten Elemente einzubringen und deutlich über die seit 1. Januar 2011 geltende Fassung hinauszugehen, die nach dem Urteil des Europäischen Gerichtshofes für Menschenrechte (EGMR) vom 17.12.2009 verabschiedet worden war.

Meine Erwartungen hinsichtlich der Beseitigung der genannten Mängel und Schwächen im Zuge des weiteren Gesetzgebungsverfahrens sind nicht sehr hoch. Es wird nun darauf ankommen, dass an den oben bezeichneten Stellen nicht noch Abschwächungen vorgenommen werden und dass die Länder ihre Teile angemessen umsetzen.

Prof. Dr. Heinz Cornel lehrt an der Alice-Salomon-Hochschule Berlin, ist Präsident des DBH-Fachverbandes für Soziale Arbeit, Strafrecht und Kriminalpolitik und Mitherausgeber der Neuen Kriminalpolitik

\section{Fußnoten}

1 Wie oben beschrieben, widersprechen die Regelungen zwar nicht der Entscheidung des Bundesverfassungsgerichts, sie weiten die Übergangszeit meines Erachtens aber unangemessen aus.

2 vgl. Entscheidung des BVerfG vom 4. Mai 2011(2 BvR 2365/09), Rn 120

\section{Ein Musterentwurf nicht ohne Wert.}

\section{Anmerkungen zum ME StVollzG1 vom Autorenkreis des AK StVollzG2}

Johannes Feest

Am 6.9.2011 haben elf Bundesländer einen gemeinsamen Musterentwurf (ME) für ein Landesstrafvollzugsgesetz vorgelegt. Der Entwurf enthält eine ganze Reihe erfreulicher Verbesserungen, leider aber auch einige Rückschritte. Die grundlegenden Schwächen, die schon das alte Gesetz zum Torso gemacht haben (Arbeitsentgelt, Krankenversicherung, Rentenversicherung) behebt er nicht. Es ist mehr als wahrscheinlich, dass die Realität des Vollzuges (zu wenig sinnvolle Angebote, einschließlich produktiver Arbeit) die normativen Fortschritte wieder zunichte machen wird. In einem rauer gewordenen sozialen Klima sind die Chancen zu einem Neuanfang für die meisten Straffälligen ohnehin miserabel.

Die Föderalisierung der Gesetzgebung zum (Erwachsenen-) Strafvollzug in Deutschland schreitet voran. Nachdem Bayern, Nie- dersachsen und Hamburg bereits 2007 gesetzgeberisch tätig geworden waren, haben im Jahre 2009 auch Baden-Württemberg und Hessen eigene Landesstrafvollzugsgesetze erlassen. Jetzt folgen die restlichen Bundesländer (mit Ausnahme Nordrhein-Westfalens), die einen gemeinsamen Musterentwurf eines Strafvollzugsgesetzes (ME) vorgelegt haben. Die Vorlage bietet Anlass zu einer kriminalpolitischen Analyse und einem Vergleich mit dem (Bundes-)Strafvollzugsgesetz (StVollzG) von 1976 sowie den bereits erlassenen Landesgesetzen, die zum Teil erhebliche Rückschritte gegenüber dem früheren Recht aufweisen ${ }^{3}$.

Anders als die bisher im Zuge der Föderalismusreform verabschiedeten Landesgesetze beschränkt sich die Gesetzesinitiative auf die Regelung des Erwachsenenstrafvollzugs ${ }^{4}$. Damit wird nicht nur den 